ARTICLE

https://doi.org/10.1038/s41467-022-28573-5

\title{
Possibility for strong northern hemisphere high- latitude cooling under negative emissions
}

Jörg Schwinger (10 ${ }^{1 凶}$, Ali Asaadi (1) ${ }^{1}$, Nadine Goris (i) ${ }^{1} \&$ Hanna Lee ${ }^{1,2}$

It is well established that a collapse or strong reduction of the Atlantic meridional overturning circulation (AMOC) would substantially cool the northern high latitudes. Here we show that there is a possibility that such cooling could be amplified under deliberate $\mathrm{CO}_{2}$ removal and result in a temporary undershoot of a targeted temperature level. We find this behaviour in Earth system models that show a strong AMOC decline in response to anthropogenic forcing. Idealized simulations of $\mathrm{CO}_{2}$ removal with one of these models indicate that the timing of negative emissions relative to $\mathrm{AMOC}$ decline and recovery is key in setting the strength of the temporary cooling. We show that the pronounced temperature-fluctuations at high northern latitudes found in these simulations would entail considerable consequences for sea-ice and permafrost extent as well as for high latitude ecosystems.

\footnotetext{
${ }^{1}$ NORCE Climate, Bjerknes Centre for Climate Research, Bergen, Norway. ${ }^{2}$ Department of Biology, Norwegian University of Science and Technology, Trondheim, Norway. ${ }^{凶}$ email: jorg.schwinger@norceresearch.no
} 
S ince the Paris agreement entered into force in 2016, global anthropogenic carbon dioxide emissions have increased by 5\% (0.58 Pg C between 2016 and 2019) ${ }^{1}$, in stark contrast to the fact that net zero $\mathrm{CO}_{2}$ emissions are required by around 2050 to meet a $50 \%$ chance of keeping the average surface temperature increase to below $1.5^{\circ} \mathrm{C}^{2}$. A temporary exceedance or overshoot of the Paris Agreement temperature targets and subsequent carbon dioxide removal (CDR) from the atmosphere ${ }^{3,4}$ is therefore increasingly discussed as an option to delay the costly transition to a zero-carbon economy and society. Indeed, climate model experiments with application of $\mathrm{CDR}^{5,6}$ support the feasibility of such a strategy, since they show that many aspects of climate change are largely reversible if certain thresholds are not crossed. On the other hand, overshoot strategies come with adverse effects related to slowly reacting components of the Earth system, for example, permafrost ${ }^{7,8}$ or the interior ocean ${ }^{9,10}$. Since it is politically attractive to delay mitigation action, it has been argued that limits of overshoot scenarios beyond which adverse effects outweigh advantages need to be investigated and defined ${ }^{11}$.

In this work, we have simulated temperature overshoots of different magnitude and duration using a state-of-the-art Earth system model (NorESM2-LM ${ }^{12,13}$, methods) based on idealized, bell-shaped emission curves that have been developed for the Zero-Emission Commitment Model Intercomparison Project $(\text { ZECMIP })^{14}$. As a reference case for our study, we take a simulation that is roughly consistent with the goals of the Paris agreement leading to a temperature increase of about $1.7^{\circ} \mathrm{C}$ in the long term with no CDR applied. This reference simulation (referred to as $\mathrm{B}^{1500}$ ) has $1500 \mathrm{Pg}$ of cumulative carbon emissions during the first 100 years and zero-emissions thereafter (Fig. 1a). Additionally, we perform three simulations with the same emission profile but higher carbon emissions $\left(\mathrm{B}^{1750}, \mathrm{~B}^{2000}\right.$, and $\mathrm{B}^{2500}$, the superscript indicating the amount of cumulative carbon emissions in Pg). These simulations reach peak temperatures between 2.2 and $3.0^{\circ} \mathrm{C}$ during the positive emission phases (Fig. $1 \mathrm{c}$ and Table 1). From these simulations, we branch off negative emission phases of 250,500, and
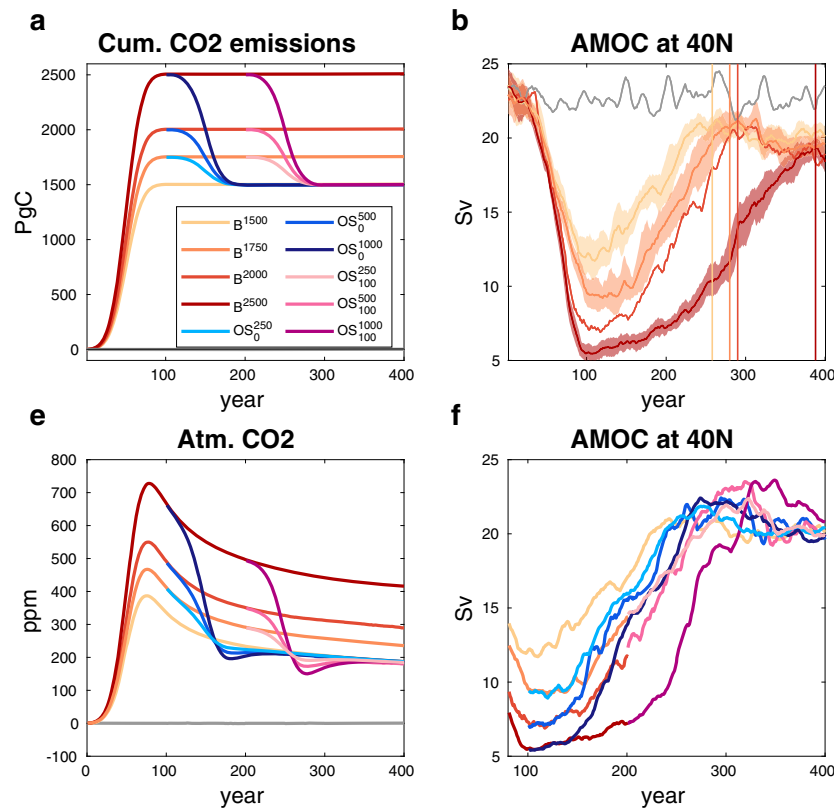

f

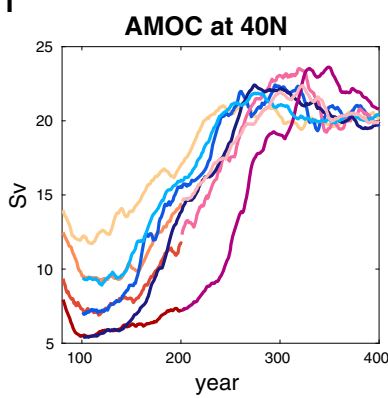

1000 Pg cumulative carbon removal, applied over a time span of 100 years. At the end of the negative emission phases, all simulations have experienced cumulative carbon emissions of $1500 \mathrm{Pg} \mathrm{C}$ (Fig. 1a). To simulate overshoots of different duration, we vary the branch point in time such that there are either 0 or 100 years of zero-emissions before CDR is applied. Thus, we get six different overshoots relative to the reference simulation, which we refer to as $\mathrm{OS}_{0}^{250}, \mathrm{OS}_{100}^{250}, \mathrm{OS}_{0}^{500}, \mathrm{OS}_{100}^{500}, \mathrm{OS}_{0}^{1000}$, and $\mathrm{OS}_{100}^{1000}$ (Table 2). Here, the superscript refers to the cumulative amount of CDR applied, and the subscript refers to the length of the zero-emission phase before the start of the simulated CDR. We stress that our simulations are idealized. For example, the phase of positive emissions is limited to 100 years, which is substantially shorter than the period from 1850 to 2100 usually covered in historical and scenario simulations with Earth system models (ESMs). In addition, we do not consider forcing from anthropogenic aerosols and greenhouse gases other than $\mathrm{CO}_{2}$. We note, however, that our simulations are closer to a realistic scenario than overshoot experiments based on a fixed rate of $\mathrm{CO}_{2}$ increase and a mirrored decrease back to preindustrial levels, which have been used extensively5,10,15-17. The latter setup results in an extremely abrupt change from positive to negative emissions, and the amount of implied negative emissions is generally huge. Our simulations have a period of increasing emissions followed by a period of decreasing emissions, and the positive and negative emission phases are smoothly joined. Further, $\mathrm{CO}_{2}$ concentrations are not returned to preindustrial levels, but to a level compatible with keeping global temperature increase well below 2 degrees. We investigate the robustness of results from our idealized model experiments by comparing with results from other types of simulations (including a more realistic scenario simulation) conducted with our model and other state-of-the-art ESMs.

\section{Results and discussion}

Positive and zero-emissions. The strength of the Atlantic meridional overturning circulation (AMOC) declines by 48 to $76 \%$ of its preindustrial strength (Fig. $1 \mathrm{~b}$ and Table 1 ) during the positive

C

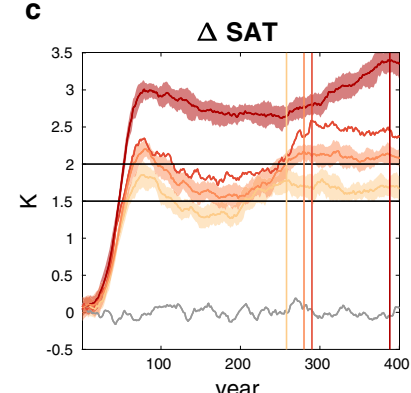

g

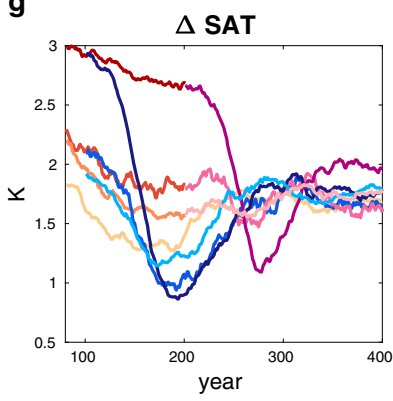

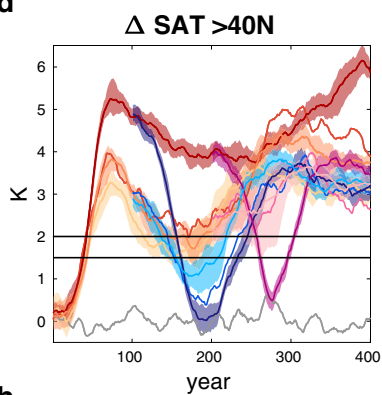

h

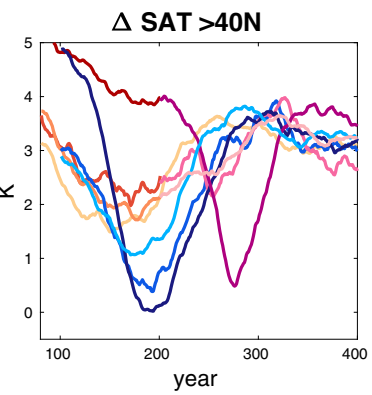

Fig. 1 AMOC and surface temperature changes in idealized NorESM2 simulations. a Cumulative carbon emissions, b, $\mathbf{f} A M O C$ strength at $40^{\circ} \mathrm{N}, \mathbf{c}, \mathbf{g}$ global average surface air temperature (SAT) change relative to preindustrial conditions, $\mathbf{d}, \mathbf{h}$ change in average SAT north of $40^{\circ} \mathrm{N}$, and $\mathbf{e}$ atmospheric $\mathrm{CO}_{2}$ concentrations for the $\mathrm{B}$ - and OS-simulations. Color coding of simulations is indicated in the legend in panel a. If available, the mean and range (shaded areas) of three ensemble members are shown. For clarity, panels $\mathbf{b}$ and $\mathbf{c}$ only show the B-simulations that have positive and zero-emissions. Likewise, panels $\mathbf{f}-\mathbf{h}$ only show the ensemble means of the overshoot simulations (blue and magenta lines) branched from the B-simulations (yellow to red lines). In panels, b-d and $\mathbf{f}-\mathbf{h}$ an 11-year running mean has been applied to the data. 


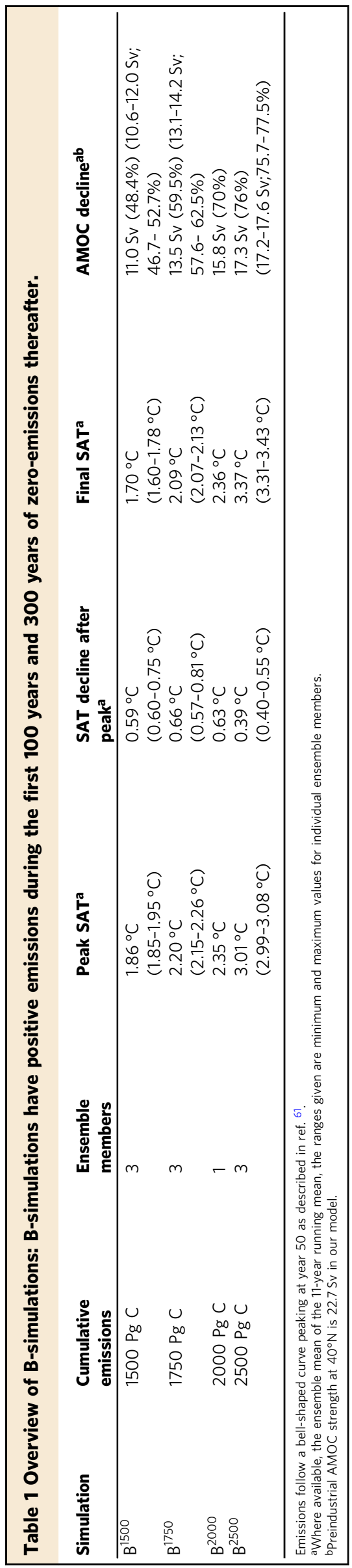

emission phases. During the zero-emission phases of the Bsimulations, AMOC strength gradually recovers and converges towards a level that is similar albeit lower by 3 to $5 \mathrm{~Sv}$ than the unperturbed state, consistent with results of a previous study ${ }^{18}$. We note that the simulated contemporary AMOC strength at $26^{\circ} \mathrm{N}$ in our model is $21 \mathrm{~Sv}$ and compares reasonably well to the observation-based estimate of $17.4 \mathrm{~Sv}^{19}$. NorESM2-LM is among the 4 CMIP6 models (disregarding one outlier model) with the highest contemporary overturning strength (CMIP6 range: 9.6 to $23 \mathrm{~Sv}$; CMIP6 mean: $17.7 \mathrm{~Sv})^{19}$. The model simulates the depth of the AMOC maximum at $0.9 \mathrm{~km}$, slightly better than the CMIP6 model mean $(0.84 \mathrm{~km})$, but still shallower than the observationbased estimate $(1.04 \mathrm{~km})^{19}$. Our model generally shows a pronounced decline of AMOC strength with climate change. We discuss this point further below, where we compare the AMOC weakening in our model to other CMIP6 models.

Global average surface air temperature (SAT) and atmospheric $\mathrm{CO}_{2}$ concentrations peak about 15 years before emissions cease (Fig. 1c, e). Thereafter, SAT declines for about a century by 0.39 to $0.66{ }^{\circ} \mathrm{C}$ (Table 1 ) before recovering and staying approximately constant until the end of our simulations (Fig. 1c; the steady-state is not reached for the $\mathrm{B}^{2500}$ simulation). The cooling during the zero-emission phase is centered around the North Atlantic (Fig. 2a) and shows the characteristic footprint of a collapsed or strongly reduced $\mathrm{AMOC}^{20-24}$. The time of SAT recovery coincides well with the recovery of AMOC strength (colored vertical lines in Fig. 1b, c). Consistent with previous studies ${ }^{21,22}$, the mechanism of the surface cooling north of $40^{\circ} \mathrm{N}$ is a strong reduction of latent heat fluxes to the atmosphere in the North Atlantic (Fig. 2c), resulting in a reduced heat loss from the ocean in this region. Atmospheric specific humidity decreases (Fig. 2e), which exerts a radiative cooling effect ${ }^{21,22}$. Since the cooling outweighs the reduced moisture flux from the ocean, low cloud cover increases (Fig. 2g), which leads to additional radiative feedback that cools the surface (Fig. S1).

Negative emissions. For this study, we take the long-term global SAT of the reference simulation $\mathrm{B}^{1500}\left(1.7^{\circ} \mathrm{C}\right.$ above preindustrial temperature) as consistent with the Paris agreement. The goal of CDR is then to lower global SAT from the levels found in the $\mathrm{B}^{1750}, \mathrm{~B}^{2000}$, and $\mathrm{B}^{2500}$ simulations to the target level of the Paris agreement. However, if net negative emissions are applied immediately after positive emissions have been phased out (i.e., in our short overshoot simulations $\mathrm{OS}_{0}^{250}, \mathrm{OS}_{0}^{500}$, and $\mathrm{OS}_{0}^{1000}$, blue lines in Fig. 1), northern high latitudes cool considerably below the target level before temperature rises again and approaches the target temperature from below (Fig. $1 \mathrm{~g}, \mathrm{~h}$ ). This effect is weak at latitudes south of $40^{\circ} \mathrm{N}$ (Fig. S2), but particularly strong north of $40^{\circ} \mathrm{N}$. Here, the $\mathrm{OS}_{0}^{1000}$ simulation even reaches preindustrial SAT levels for a short period of time before bouncing up to the reference level, which is $\sim 3^{\circ} \mathrm{C}$ above preindustrial temperature for this latitude band.

These results suggest that northern high-latitude cooling that is seen when emissions are phased out and that is caused by a strong reduction of AMOC can be amplified if CDR is applied subsequently. The mechanisms and feedbacks leading to this amplification are the same as explained above for the B-simulations (Fig. 2 and Fig. S1, left and right columns of panels). However, the negative emission phases of the overshoot simulations start from a state with a weaker AMOC compared to the reference simulation, since a larger amount of positive emissions leads to a stronger AMOC-reduction. The combination of a weaker AMOC state and a reduction of the radiative forcing by CDR amplifies the northern high-latitude cooling that is already present in the reference simulation. 
Table 2 Simulation overview for the negative emission simulations.

\begin{tabular}{llllll} 
Simulation & Parent simulation & $\begin{array}{l}\text { Cumulative positive } \\
\text { emissions }\end{array}$ & Cumulative CDR & $\begin{array}{l}\text { Time between positive and negative } \\
\text { emissions }\end{array}$ & Ensemble members \\
\hline $\mathrm{OS}_{0}^{250}$ & $\mathrm{~B}^{1750}$ & $1750 \mathrm{Pg} \mathrm{C}$ & $250 \mathrm{Pg} \mathrm{C}$ & 0 years & 3 \\
$\mathrm{OS}_{100}^{250}$ & $\mathrm{~B}^{1750}$ & $1750 \mathrm{Pg} \mathrm{C}$ & $250 \mathrm{Pg} \mathrm{C}$ & 100 years & 3 \\
$\mathrm{OS}_{0}^{500}$ & $\mathrm{~B}^{2000}$ & $2000 \mathrm{Pg} \mathrm{C}$ & $500 \mathrm{Pg} \mathrm{C}$ & 0 years & 1 \\
$\mathrm{OS}_{100}^{500}$ & $\mathrm{~B}^{2000}$ & $2000 \mathrm{Pg} \mathrm{C}$ & $500 \mathrm{Pg} \mathrm{C}$ & 100 years & 1 \\
$\mathrm{OS}_{0}^{1000}$ & $\mathrm{~B}^{2500}$ & $2500 \mathrm{Pg} \mathrm{C}$ & $1000 \mathrm{Pg} \mathrm{C}$ & 0 years & 3 \\
$\mathrm{OS}_{100}^{1000}$ & $\mathrm{~B}^{2500}$ & $2500 \mathrm{Pg} \mathrm{C}$ & $1000 \mathrm{Pg} \mathrm{C}$ & 100 years & 3 \\
\hline
\end{tabular}

a

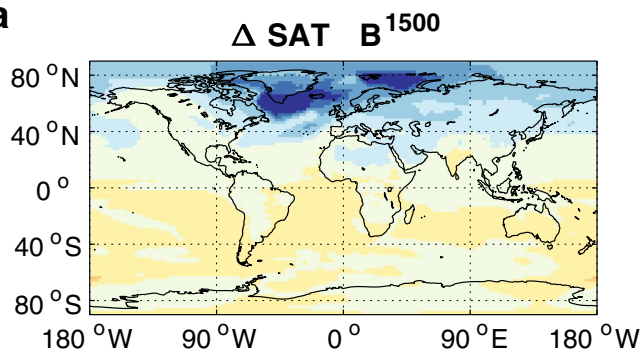

C

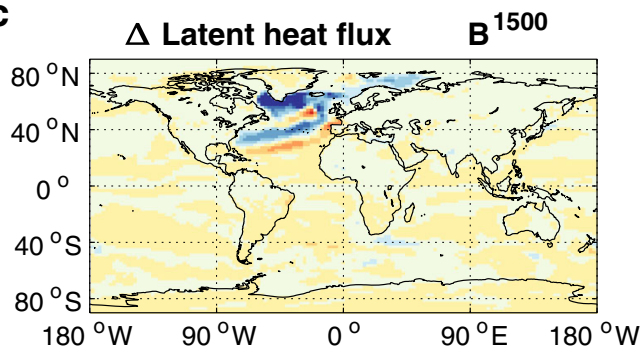

e

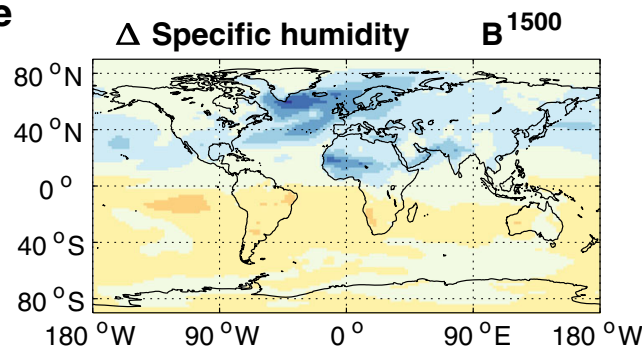

g

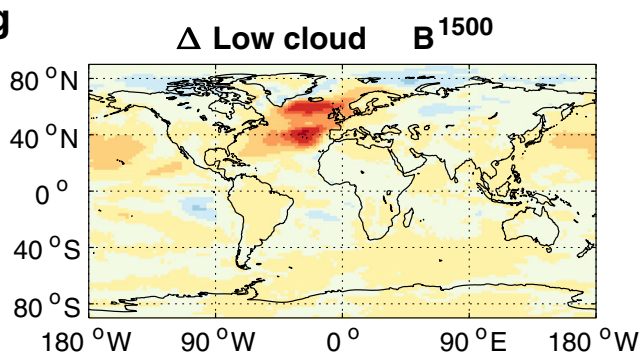

b
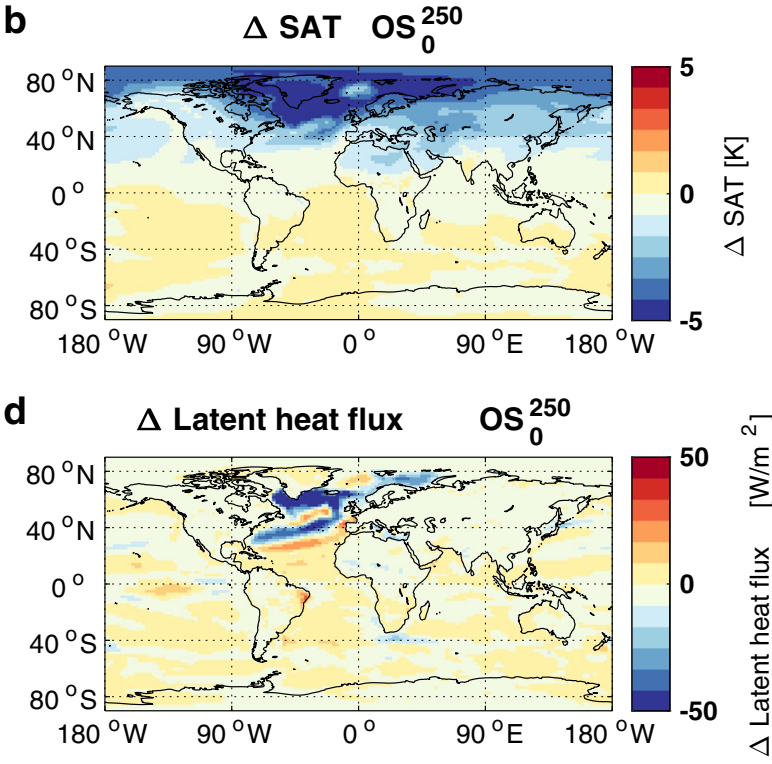

f

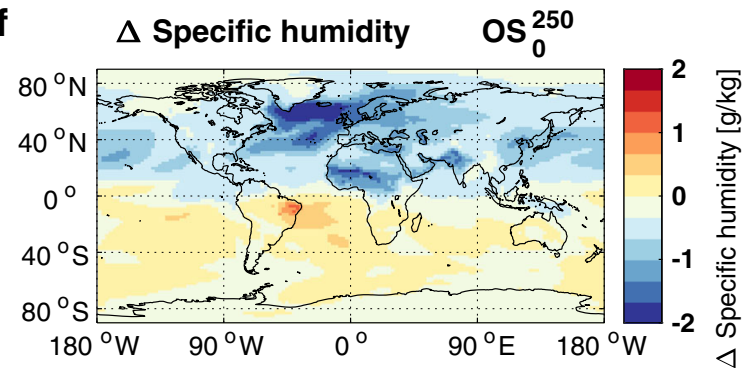

h

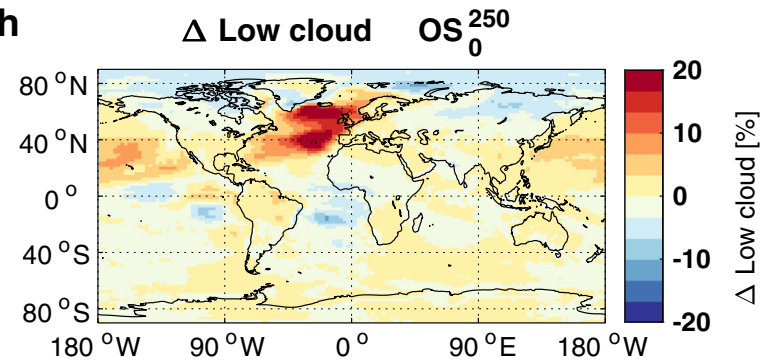

Fig. 2 Impacts of AMOC decline under zero and negative emissions. Difference between a state with reduced AMOC around the SAT minimum (years 180-190) and a state with recovered AMOC and SAT (years 280-289) for the reference simulation $B^{1500}$ (left column) and the OS ${ }_{0}^{250}$ overshoot (right column). Shown is the mean of three ensemble members for $\mathbf{a}, \mathbf{b}$ SAT, $\mathbf{c}, \mathbf{d}$ latent heat flux, e, $\mathbf{f}$ specific humidity, and $\mathbf{g}$, $\mathbf{h}$ low cloud cover.

Further analysis of the temperature response north of $40^{\circ} \mathrm{N}$ indicates that absolute temperature anomalies (relative to preindustrial temperature) in the overshoot simulations remain smaller over the ocean than over land, with a particularly strong cooling over the North Atlantic (Fig. S3a-c), as expected from the strong reduction of AMOC strength. Absolute surface temperature anomalies in Europe are the smallest of all continents due to the proximity and downwind location relative to the North Atlantic. However, our analysis also shows that the additional cooling in the overshoot simulations relative to the reference (i.e., the simulation without overshoot) is regionally very similar north of $40^{\circ} \mathrm{N}$ (Fig. S3d-f). There is a tendency that 
land masses located further downwind from the source of the anomaly in the North Atlantic experience less additional cooling during the overshoot, i.e. the additional cooling is weaker in North America than in Asia and weaker in Asia than in Europe. This effect can be clearly seen for the highest overshoot, but it is less pronounced in the low overshoot case (Fig. S3a, d). This indicates that the amplified cooling north of $40^{\circ} \mathrm{N}$ during an overshoot in our model is spatially very coherent with only minor regional differences.

If negative emissions are applied later, after 100 years of zeroemissions (Fig. 1, magenta lines), when AMOC strength is already recovering, the cooling effect of $\mathrm{CDR}$ is much weaker or even absent. In the $\mathrm{OS}_{100}^{250}$ and $\mathrm{OS}_{100}^{500}$ simulations, CDR starts at a point in time when AMOC strength has recovered to more than $50 \%$ of its original strength and SAT north of $40^{\circ} \mathrm{N}$ increases in the B-simulations (from which the overshoots are branched off) as well as in the reference simulation. In these cases, CDR mainly mitigates the increase of temperature rather than leading to cooling. Only the $\mathrm{OS}_{100}^{1000}$-overshoot still shows a substantial cooling, consistent with the fact that its associated AMOC recovery is slower compared to the other simulations and SAT is still decreasing when CDR is applied.

Impacts and challenges for adaptation. If CDR is applied to lower surface air temperature, then why would we care if regional temperature undershoots the target level for some time, and could we not avoid such undershoot by regulating the pace of $\mathrm{CO}_{2}$ removal? For the medium-sized overshoot $\mathrm{OS}_{0}^{500}$, we find that the maximum cooling below the target level during the phase of negative emissions is $-1.79,-0.31$, and $-0.12^{\circ} \mathrm{C}$, north of $40^{\circ} \mathrm{N}$, between $40^{\circ} \mathrm{S}$ to $40^{\circ} \mathrm{N}$, and south of $40^{\circ} \mathrm{S}$, respectively (Fig. S2). These results illustrate that there could be tradeoffs or conflicts between the global north and south in a scenario of CDR deployment, specifically when avoiding a temperature undershoot below the target level in the north would require delaying CDR and accepting climate impacts in the south for a prolonged period of time. This is nicely illustrated by the long variants of the low and medium overshoots $\left(\mathrm{OS}_{100}^{250}\right.$ and $\left.\mathrm{OS}_{100}^{500}\right)$ which provide a quite smooth temperature trajectory (with minimal undershoot) towards the reference simulation in the northern high latitudes and would certainly be considered an optimal CDR strategy for this region in isolation. Here, one would leverage the northern high-latitude SAT decline due to reduced AMOC strength until the recovery of AMOC increases SAT, which would then be offset by negative emissions. However, such a strategy would come at the cost of accepting elevated temperatures south of $40^{\circ} \mathrm{N}$ for an extended period of time.

An AMOC slowdown or collapse is thought to have severe impacts globally. Most notably, a southward shift of tropical rainfall and an increase of droughts in the Amazon and Sahel regions ${ }^{25}$ would affect a large and vulnerable population. Here, we focus on the high-latitude temperature fluctuations seen in our overshoot simulations noting that the AMOC slowdown itself is in fact mitigated by the application of CDR in our model (i.e., AMOC recovery happens faster with than without CDR).

What would be the consequences of temperature fluctuations of the magnitude seen in the $\mathrm{OS}_{0}^{250}$ and $\mathrm{OS}_{0}^{500}$ simulations? The impacts of climate change on ecosystems and economies, as well as challenges for adaptation, are often discussed in terms of increasing global mean temperatures only. We anticipate that the northern high-latitude temperature trajectories in our overshoot simulations with their pronounced warming-cooling-warming pattern would most likely exacerbate impacts and challenges ${ }^{26}$. To illustrate this point, we pick three examples from our ESM simulations (Fig. 3).
Arctic summer sea-ice is melting rapidly during the positive emission phases, resulting in a sea-ice-free Arctic during September (less than 1 million square $\mathrm{km}$ covered by sea-ice) in all B-simulations (Fig. S4). During the zero-emission phases summer sea-ice-cover follows the trends in SAT, leading to recovery until the SAT minimum is reached and a renewed decline thereafter. If CDR is applied, the amplified northern highlatitude cooling has a pronounced impact on the Arctic summer sea-ice extent (Fig. S4). Even in our low overshoot simulation, $72 \%$ of the area of the preindustrial Arctic summer sea-ice extent undergoes a thaw-refreeze-rethaw cycle within 200 years (methods, Fig. 3a). For comparison, this area is only $42 \%$ in the reference simulation and $85 \%(100 \%)$ in the $\mathrm{OS}_{0}^{500}\left(\mathrm{OS}_{0}^{1000}\right)$ overshoot. The retreat of sea-ice and changing environmental conditions will lead to gradual or rapid shifts in Arctic ecosystems through adaptation, migration, or extinction of species ${ }^{27-30}$, and in general, such shifts are not easily reversible ${ }^{31}$. Therefore, a period of cooling after a warming-induced ecosystem shift might (instead of just reversing the shift) act as an additional stressor to the ecosystem and cause further loss of biodiversity. On the other hand, the retreat of Arctic sea-ice also brings economic opportunities in mining, gas and oil exploration ${ }^{32}$, tourism ${ }^{33}$, fishing ${ }^{34}$, and shipping ${ }^{35}$. A pronounced but intermittent cooling with regrowth of sea-ice can be expected to have negative economic impacts, for example by closing newly opened Arctic shipping routes or by making large Arctic regions inaccessible for economic exploitation again. This will either hinder economic development (if the cooling is correctly predicted) or lead to stranded assets (if the cooling comes as a surprise).

Similar to summer sea-ice, the permafrost extent declines rapidly in response to warming and follows the SAT trend very closely afterward (Fig. S5). Consequently, permafrost thaws, refreezes, and thaws again over considerable areas in our overshoot scenarios (Fig. 3b). The extent of an area undergoing such cycles is $12,17.7$, and $20.9 \%$ of the preindustrial permafrost extent $\left(15.3 \times 10^{6} \mathrm{~km}^{2}\right.$ in our model $)$ in the $\mathrm{OS}_{0}^{250}, \mathrm{OS}_{0}^{500}$, and $\mathrm{OS}_{0}^{1000}$ simulations, respectively. Again, the area affected by thawing, refreezing, and thawing is significantly smaller in the reference simulation without CDR (7.8\%). These results are consistent with a previous modeling study that suggests global scale permafrost degradation trajectories will highly depend on temperature, and the physical state of permafrost is reversible under global scale temperature reduction ${ }^{36}$. Given that nearly 5 million people inhabit permafrost areas ${ }^{37}$, and different types of human infrastructure are built on permafrost (including transportation networks, mining projects, electrical power transmission lines, and water retaining structures as well as general buildings), permafrost thaw will entail considerable costs related to adapting infrastructure to the changing environmental conditions (e.g., ground subsidence, bearing capacity of the ground, uneven surface deformation ${ }^{38}$. Several studies have provided quantitative assessments of potential economic impacts of permafrost thaw on various types of infrastructures ${ }^{38-40}$. Engineering solutions for permafrost environments are generally specialized, for example, ref. ${ }^{39}$. could not identify adaptation measures for permafrost thaw that were less expensive than complete infrastructure replacement. This might indicate that permafrost thaw-refreeze-rethaw cycles could significantly increase adaptation costs if permafrost thaw occurs twice during the nominal lifetime of human infrastructure.

The potential growth of farmed and wild Atlantic Salmon (Salmo Salar) is confined to a thermal window, ranging from optimal conditions around $14^{\circ} \mathrm{C}$ to lethal conditions below $1.5^{\circ} \mathrm{C}$ or above $19^{\circ} \mathrm{C}^{41}$. In our overshoot simulations, the cycle of North Atlantic SST warming, cooling, and subsequent warming results 
a

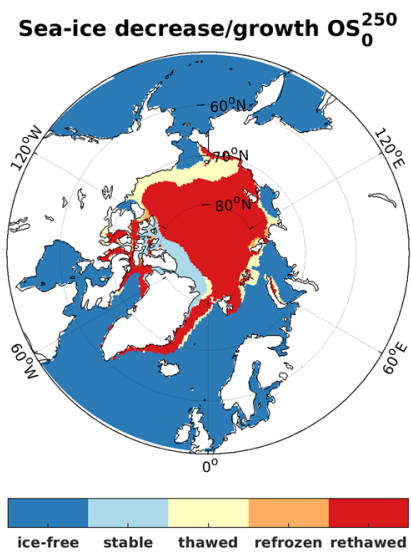

b

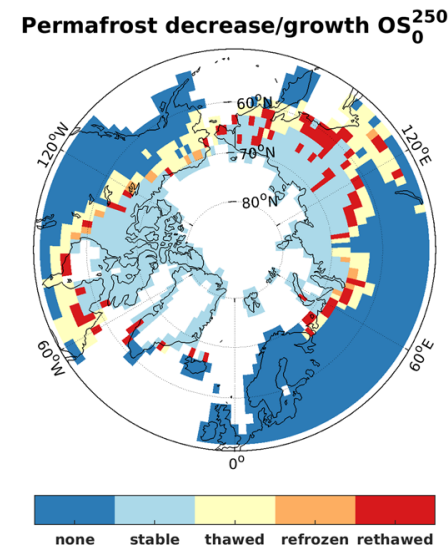
Potential Salmon growth $0 \mathrm{~s}_{0}^{250}$
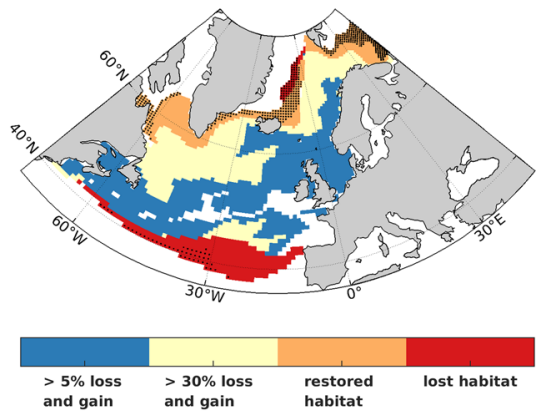

Fig. 3 Impacts of warming-cooling-warming cycles. Evolution of northern hemisphere a summer sea-ice, $\mathbf{b}$ permafrost, and $\mathbf{c}$ Atlantic salmon tolerable temperature range under the $\mathrm{OS}_{0}^{250}$ overshoot simulation. In a and $\mathbf{b}$, the light blue color indicates stable conditions, yellow color indicates permanent thaw, orange color indicates areas where thaw and refreezing occurred consecutively, and red areas indicate that a thaw-freeze-thaw cycle occurred (methods). In panel c, potential salmon growth rate loss and gain between 5 and $30 \%$ is indicated in blue, between 30 and $100 \%$ in yellow, and between lost and restored habitat in orange (methods). Habitat that is lost between SAT minimum and SAT recovery is marked in red. Stripeling indicates habitat gained during the final warming stage. Corresponding figures for the reference simulation and the $0 S_{0}^{500}$ and $O S_{0}^{1000}$ overshoots can be found in the supplementary material (Figs. S6-S8).

in optimal growth conditions shifting northward, then southward, and finally northward again. As a consequence, in the $\mathrm{OS}_{0}^{250}$ simulation, the potential growth rate undergoes alternating positive and negative changes with $40 \%$ of the preindustrial habitat being exposed to changes in the potential growth rate of $5-30 \%$ during each shift, $29 \%$ of the habitat to changes of $30-100 \%$, and $26 \%$ of the habitat is lost and partly restored within shifts (Fig. 3c). In the reference run without overshoot, the corresponding changes in area are smaller and only $20 \%$ of the original habitat is lost and partly restored, while it is $36 \%$ in $\mathrm{OS}_{0}^{500}$ and $40 \%$ in $\mathrm{OS}_{0}^{1000}$. Such changes could have considerable economic consequences as farmed Atlantic Salmon is the most successful aquaculture species with the highest productivity in the northern hemisphere ${ }^{42}$ and the exposed regions comprise areas with a high density of coastal aquaculture installations (Norway, Scotland, Faroe Island, and Canada $)^{42}$, as well as areas for emerging offshore aquaculture in Norway ${ }^{43}$. Ecological consequences for wild Atlantic Salmon could also be expected. Stocks of this migrating species have been declining over the last century due to anthropogenic stressors ${ }^{44}$, and fluctuating temperature conditions along the natal rivers and migratory pathways will constitute an additional stressor and threaten the survival of an already vulnerable species.

Robustness of cooling response. How robust are the results of our simulations? To compare the behavior of our model with other ESMs we use two different simulations from the CMIP6 archive. The first one is a highly idealized simulation of CDR (1pctCO2-cdr) available through the carbon dioxide removal intercomparison project (CDRMIP) ${ }^{17}$. This simulation extends the standard experiment where atmospheric $\mathrm{CO}_{2}$ is increased by $1 \%$ per year until quadrupling after 140 years, with a mirrored simulation where $\mathrm{CO}_{2}$ is decreased by $1 \%$ per year until preindustrial concentrations have been restored after 280 years. In addition to NorESM2-LM, seven CMIP6 ESMs ${ }^{45-51}$ have performed this simulation (Fig. 4a-c). Three models (CESM2, CNRM-ESM-2, and GFDL-ESM4) show initially a strong AMOC $\left(>20 \mathrm{~Sv}\right.$ at $\left.40^{\circ} \mathrm{N}\right)$ and a strong decline $(>10 \mathrm{~Sv})$, similar to NorESM2-LM, which has the second strongest decline in absolute numbers (Fig. 4b). In the North Atlantic (between $47^{\circ} \mathrm{N}$ and $80^{\circ} \mathrm{N}$ ), these models show a pronounced decline of surface temperatures below the preindustrial level towards the end of the $1 \%$-ramp down phase (Fig. 4c). One model (MIROC2-ES2L) that has a weaker initial AMOC strength and lesser decline also shows SAT below the preindustrial level in this region. The spatial cooling pattern is quite similar between the models with strong AMOC decline (Fig. S9). There is, however, also a dependence on how fast AMOC strength recovers from its minimum value, and on the intensity of global warming (i.e., on the transient climate response). For example, while the GFDL-ESM4 and CNRM-ESM2 models show a very similar decline in AMOC strength, recovery of AMOC is much faster in GFDL-ESM4. Therefore, the cooling is less pronounced in the latter model, since at the time the radiative forcing reaches preindustrial levels (year 280 of the simulation), AMOC strength has already completely recovered. NorESM2-LM has the lowest transient global warming of the eight ESMs, and therefore it initially cools faster than CESM2 which shows a similar decline in AMOC strength. We note that NorESM2 is based on CESM2 but implements a different ocean model. Therefore, the strong cooling seen in CESM2 indicates that this phenomenon is not a peculiarity of our ocean model.

The second simulation that we investigate is the ScenarioMIP52 SSP5-3.4-overshoot scenario, for which output from nine models ${ }^{46-50,53-55}$ (including NorESM2-LM) is available. In contrast to our idealized experiments and to the $1 \mathrm{pctCO} 2-\mathrm{cdr}$ simulation, this scenario has been created by an integrated assessment model and represents a plausible emission pathway with an unmitigated growth of emissions until 2040 and strong mitigation including negative emissions afterward. In the ensemble of nine ESMs, five models simulate a weaker and three models a stronger AMOC reduction by 2100 compared to NorESM2-LM (Fig. 4d, e). Four ESMs including our model show a decline of AMOC strength of more than $50 \%$ during the positive emission phase of this scenario. NorESM2-LM and two others of these models (CESM2-WACCM and MRI-ESM2) show a pronounced cooling below preindustrial levels in the NorthAtlantic sector during the negative emission phase. Interestingly, these three models are the same models that exhibit rapid cooling events in the subpolar gyre region in some of the CMIP6 scenario simulation $^{56}$. One model with pronounced AMOC reduction (GISS-E2-1-G) also shows a cooling trend in the North Atlantic sector, but the cooling is less pronounced (SAT does not fall 

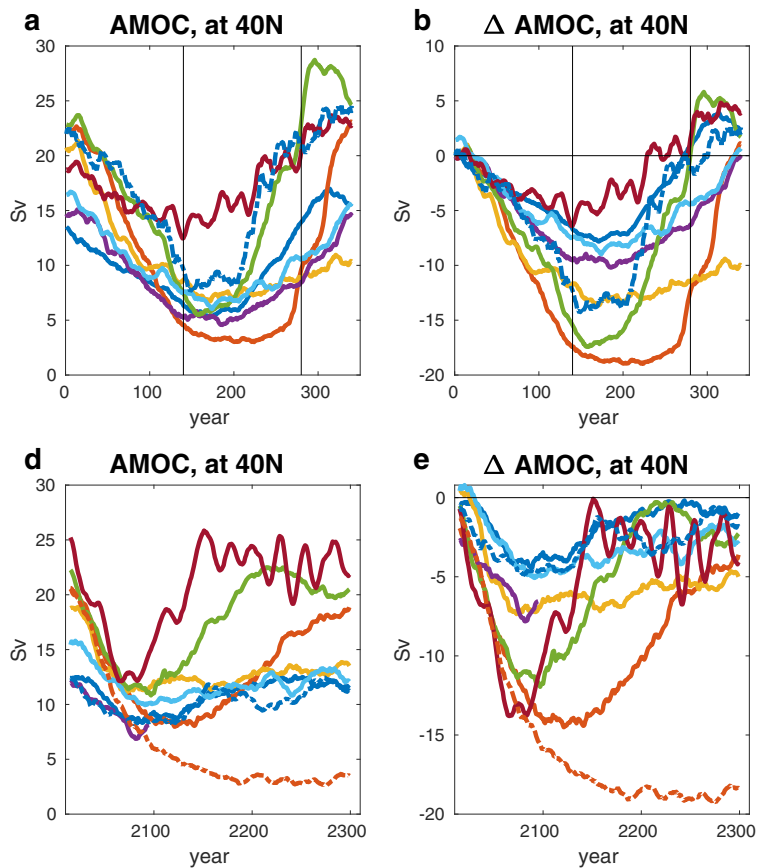

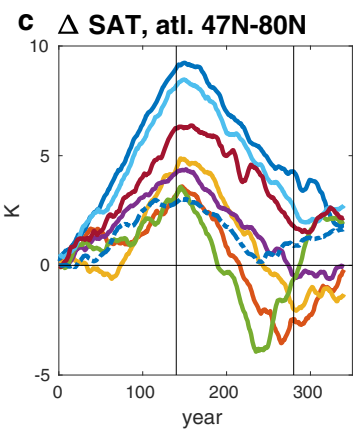

f $\triangle$ SAT, atl. $47 \mathrm{~N}-80 \mathrm{~N}$

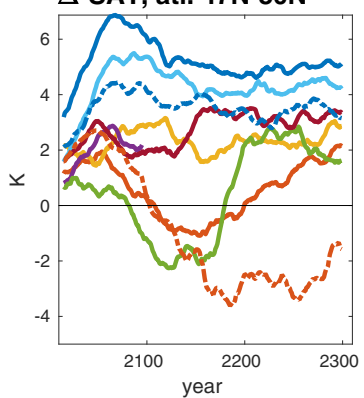

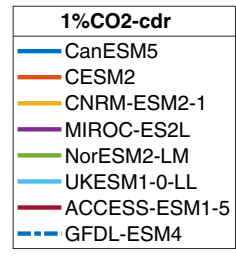

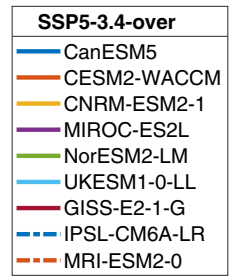

Fig. 4 Northern high-latitude cooling in CDRMIP and ScenarioMIP simulations. Results from the multi-model intercomparison projects CDRMIP and ScenarioMIP for $\mathbf{a}, \mathbf{d}$ AMOC strength at $40^{\circ} \mathrm{N}, \mathbf{b}$, e change in AMOC strength relative to the preindustrial control simulation, and $\mathbf{c}, \mathbf{f}$ change in SAT over the North Atlantic between 47 and $80^{\circ} \mathrm{N}$. Panels a-c show results from the CDRMIP reversibility experiment (1pctCO2-cdr), where thin vertical lines mark the transition from $1 \% \mathrm{CO}_{2}$ increase per year to $1 \%$ decrease at year 140 , and the transition from $1 \% \mathrm{CO}_{2}$ decrease to constant preindustrial $\mathrm{CO}_{2}$ at year 280. Panels $d-f$ show results for the ScenarioMIP SSP5-3.4-over simulation.

below preindustrial levels) due to a faster AMOC recovery in this model. In NorESM2-LM, SAT in the North Atlantic sector drops below preindustrial levels towards the end of the 21st century and reaches a minimum of $2^{\circ}$ below preindustrial SAT around 2120 . Thereafter, SAT stays at this low level for about 50 years before quickly bouncing up to more than $2^{\circ}$ above preindustrial SAT as AMOC recovers. The magnitude and timescale of the SAT response in the North Atlantic sector between $47^{\circ}$ and $80^{\circ} \mathrm{N}$ simulated by our model for the SSP5-3.4 scenario is consistent with the SAT response in our idealized $\mathrm{OS}_{0}^{250}$ overshoot simulation (compare Fig. S3c). These results confirm that AMOC decline and northern high-latitude cooling in our model is within the range of results of other CMIP6 Earth system models and that the amplification of cooling in our idealized NorESM2-LM simulations is not significantly different compared to more realistic scenarios.

We note that some models show a strengthening of AMOC above preindustrial levels after the atmospheric $\mathrm{CO}_{2}$ concentration has been returned to preindustrial levels in the CDRMIP 1 pctCO2-cdr experiment (Fig. $4 \mathrm{~b}$ ). In our model, this leads to high-latitude warming following the cooling period (Fig. 4c). Such high-latitude warming has been described in earlier studies ${ }^{15,16}$, which used a similar experimental setup, and acts to reinforce the warming-cooling-warming cycle described in this study. However, an acceleration of AMOC after a period of negative emissions seems to be a characteristic of simulations with extremely strong forcing such as the $1 \mathrm{pctCO} 2$-cdr experiment. There is no strengthening of AMOC above preindustrial levels in the SSP5-3.4 scenario simulation in any of the models, and we do not observe this in our idealized simulation either. In summary, these results demonstrate that a strong North Atlantic cooling under negative emissions seems to be a robust feature of Earth system models that show a strong and sustained AMOC decline in response to warming.
Uncertainties. Our study shows that phasing out $\mathrm{CO}_{2}$ emissions could lead to a significant centennial cooling trend relative to peak temperatures north of $40^{\circ} \mathrm{N}$ if the preceding global warming has strongly weakened the Atlantic meridional overturning circulation. If net negative emissions are applied in a state of a strongly weakened AMOC, the temporary northern high-latitude cooling can be amplified, leading to a pronounced warming-cooling-warming pattern in overshoot scenarios. The ESM used in this study (NorESM2) shows a relatively strong decline of AMOC strength but is not an outlier compared to other state-of-the-art ESMs. Our simulations are idealized and the identified consequences for seaice, permafrost and ecosystems might not be directly transferable to more realistic mitigation scenarios. We do find, however, a similar pattern of northern high-latitude temperature fluctuations in a simulation of a plausible future scenario with net negative emissions. There is considerable uncertainty regarding the future fate of the AMOC, and this has been identified as one of the major sources of uncertainty in climate projections $\mathbf{s}^{23,24,57}$. We are currently unable to assess how strongly and abruptly AMOC strength will change under climate warming and how fast the circulation might recover, but it has been suggested that ESMs, in general, tend to underestimate the possibility for abrupt and strong changes 58 , which, in the case of AMOC, might be related to common biases ${ }^{59}$. Also, the melting of the Greenland ice sheet, which might further destabilize AMOC particularly on longer time scales $^{60}$ is not included in our and other state-of-the-art ESMs. Given the considerable impacts of the northern high-latitude cooling highlighted in our study, reducing the uncertainty of AMOC projections should be pursued with high priority.

\section{Methods}

NorESM simulations. We use the Norwegian Earth System Model (NorESM2$\mathrm{LM})^{12,13}$ in emission-driven configuration, that is, $\mathrm{CO}_{2}$ is emitted into the atmosphere where it is advected with the atmospheric circulation and taken up or 
released by the land biosphere and the ocean. $\mathrm{CO}_{2}$ emissions are constructed following the protocol of the Zero-Emission Commitment Model Intercomparison Project (ZECMIP) ${ }^{61}$ with total emissions of $1500,1750,2000$, and $2500 \mathrm{Pg}$ C over 100 years. Spatially, emissions are distributed uniformly over the sphere. Negative emissions for totals of 250,500 , and $1000 \mathrm{Pg} \mathrm{C}$ are constructed in the same way, but with a negative sign. No other forcing is varied in the model, that is, land use as well as non- $\mathrm{CO}_{2}$ greenhouse gas and aerosol emissions and concentrations stay at preindustrial levels. For all simulations except the $\mathrm{B}^{2000}$ and medium-sized overshoots $\mathrm{OS}_{0}^{500}$ and $\mathrm{OS}_{100}^{500}$, we have run three ensemble members. We note that the climate sensitivity of NorESM2 is at the low end when compared to other CMIP6 models ${ }^{62}$ and that most other models would show more warming for these levels of cumulative $\mathrm{CO}_{2}$ emissions.

Cumulative emissions of $2500 \mathrm{Pg} \mathrm{C}$ are comparable to the $\mathrm{CO}_{2}$ emissions of the Shared Socioeconomic Pathway (SSP) SSP5-8.5 (2637 $\pm 136 \mathrm{Pg} \mathrm{C})^{63}$, while the $1500 \mathrm{Pg}$ C emissions of the reference case correspond to SSP4-6.0 emissions (1498 \pm 80 Pg C). For comparison, emissions over the historical period from 1850 to 2019 are estimated at $650 \pm 65 \mathrm{Pg} \mathrm{C}^{1}$. Scenarios that are consistent with limiting global warming to $1.5^{\circ} \mathrm{C}$ until 2100 contain up to $330 \mathrm{Pg} \mathrm{C}$ negative emissions ${ }^{2}$, although the uncertainties surrounding such estimates are large (e.g., ref. ${ }^{3}$ ). Also, the quoted $330 \mathrm{Pg} \mathrm{C}$ are gross negative emissions, and part of this carbon dioxide removal might be used to compensate for residual, difficult to mitigate positive emissions, and the amount of net negative emissions in these scenarios will be somewhat lower. Nevertheless, the amount of CDR in our low overshoot cases (250 Pg C) is clearly within the range of CDR discussed in the current scenario literature, while the amount of CDR applied in the medium overshoots $(500 \mathrm{Pg} \mathrm{C})$ is higher than this range, but still could be considered roughly consistent with IPCC scenarios given the longer timescale of our simulations. The amount of CDR applied in the high overshoot cases (1000 Pg C) most likely falls outside a feasible range. Here, we focus on the low and medium overshoot cases, but we include the results of the high overshoot simulations since they complement our understanding of the Earth system response to different levels of CDR.

Sea-ice thaw-refreeze-rethaw cycles. We define thaw of sea-ice if the September fractional ice-cover in a given grid cell over the first 11 years of the overshoot simulations changed by more than $50 \%$ relative to a 30 -year preindustrial climatology. Refreeze is defined as an increase of September fractional ice-cover by $50 \%$ (or by an absolute increase of 0.3 ) during the sea-ice maximum in the negative emission phases relative to the first 11 years of the overshoots. Rethaw is defined as a thaw of previously refrozen grid points during the years 300-310 of the simulations when SAT and September sea-ice area become very similar in the reference and all short overshoot simulations.

Permafrost thaw-refreeze-rethaw cycles. We define permafrost grid points where the maximum active layer thickness is below $3 \mathrm{~m}$. As for sea-ice, the thawed permafrost area is calculated for the first 11 years of the overshoot simulations relative to a 30-year preindustrial climatology. Refreeze is defined as the increase in permafrost area during the permafrost maximum in the negative emission phases relative to the first 11 years of the overshoots. Rethawed area is calculated for the years $300-310$ of the simulations relative to the previous maximum.

Potential salmon growth. For calculation of potential salmon growth, we follow the thermal performance curve of ref. ${ }^{41}$. These authors specify the somatic growth of salmon ( $\mathrm{G}, \mathrm{kg}$ per month) as a piecewise linear function that is dependent on sea surface temperature (SST, in ${ }^{\circ} \mathrm{C}$ ): $\mathrm{G}(\mathrm{SST})=0.0264 \times \mathrm{SST}-0.0396$ for SST $<14$ and $\mathrm{G}(\mathrm{SST})=-0.066 \times \mathrm{SST}+1.254$ for SST $\geq 14$. At temperatures below the minimum temperature for growth $\left(1.5^{\circ} \mathrm{C}\right)$ and above the maximum temperature for growth $\left(19^{\circ} \mathrm{C}\right), \mathrm{G}$ is set to zero to reflect the absence of growth. If for a given grid point, $\mathrm{G}$ is zero over the whole decade, we consider this grid point as non-habitable for Salmon.

\section{Data availability}

The model data generated in this study are available through the Norwegian Research Data Archive/Bjerknes Climate Data Centre and can be accessed under https://doi.org/ 10.11582/2022.00012. The CMIP6 model output data used in this study are available from the Earth System Grid Federation (ESGF) servers under https://esgf.llnl.gov.

\section{Code availability}

The source code of NorESM2 is available at https://doi.org/10.5281/zenodo.3905091

Received: 16 July 2021; Accepted: 24 January 2022;

Published online: 01 March 2022

\section{References}

1. Friedlingstein, P. et al. Global Carbon Budget 2020. Earth Syst. Sci. Data 12, 3269-3340 (2020).

2. Masson-Delmotte, V. et al. Global Warming of $1.5^{\circ} \mathrm{C}$ (IPCC, 2018).
3. Fuss, S. et al. Negative emissions-Part 2: costs, potentials and side effects. Environ. Res. Lett. 13, 063002 (2018).

4. Keller, D. P. et al. The effects of carbon dioxide removal on the carbon cycle. Curr. Clim. Change Rep. 4, 250-265 (2018).

5. Boucher, O. et al. Reversibility in an earth system model in response to $\mathrm{CO} 2$ concentration changes. Environ. Res. Lett. 7, 024013 (2012).

6. Tokarska, K. B., Zickfeld, K. \& Rogelj, J. Path independence of carbon budgets when meeting a stringent global mean temperature target after an overshoot. Earths Future 7, 1283-1295 (2019).

7. Comyn-Platt, E. et al. Carbon budgets for 1.5 and $2{ }^{\circ} \mathrm{C}$ targets lowered by natural wetland and permafrost feedbacks. Nat. Geosci. 11, 568-573 (2018).

8. MacDougall, A. H., Zickfeld, K., Knutti, R. \& Matthews, H. D. Sensitivity of carbon budgets to permafrost carbon feedbacks and non-CO 2 forcings. Environ. Res. Lett. 10, 125003 (2015).

9. Mathesius, S., Hofmann, M., Caldeira, K. \& Schellnhuber, H. J. Long-term response of oceans to $\mathrm{CO} 2$ removal from the atmosphere. Nat. Clim. Change 5, 1107-1113 (2015).

10. Schwinger, J. \& Tjiputra, J. Ocean carbon cycle feedbacks under negative emissions. Geophys. Res. Lett. 45, 5062-5070 (2018).

11. Geden, O. \& Löschel, A. Define limits for temperature overshoot targets. Nat. Geosci. 10, 881-882 (2017).

12. Seland, Ø. et al. Overview of the Norwegian Earth System Model (NorESM2) and key climate response of CMIP6 DECK, historical, and scenario simulations. Geosci. Model Dev. 13, 6165-6200 (2020).

13. Tjiputra, J. F. et al. Ocean biogeochemistry in the Norwegian Earth System Model version 2 (NorESM2). Geosci. Model Dev. 13, 2393-2431 (2020).

14. MacDougall, A. H. et al. Is there warming in the pipeline? A multi-model analysis of the Zero Emissions Commitment from $\mathrm{CO}_{2}$. Biogeosciences 17, 2987-3016 (2020).

15. Wu, P., Jackson, L., Pardaens, A. \& Schaller, N. Extended warming of the northern high latitudes due to an overshoot of the Atlantic meridional overturning circulation. Geophys. Res. Lett. 38, L24704 (2011).

16. Wu, P., Ridley, J., Pardaens, A., Levine, R. \& Lowe, J. The reversibility of $\mathrm{CO} 2$ induced climate change. Clim. Dyn. 45, 745-754 (2015).

17. Keller, D. P. et al. The carbon dioxide removal model intercomparison project (CDRMIP): rationale and experimental protocol for CMIP6. Geosci. Model Dev. 11, 1133-1160 (2018).

18. Sigmond, M., Fyfe, J. C., Saenko, O. A. \& Swart, N. C. Ongoing AMOC and related sea-level and temperature changes after achieving the Paris targets. Nat. Clim. Change 10, 672-677 (2020).

19. Weijer, W., Cheng, W., Garuba, O. A., Hu, A. \& Nadiga, B. T. CMIP6 models predict significant 21 st century decline of the Atlantic meridional overturning circulation. Geophys. Res. Lett. 47, e2019GL086075 (2020).

20. Vellinga, M. \& Wood, R. A. Global climatic impacts of a collapse of the atlantic thermohaline circulation. Clim. Change 54, 251-267 (2002).

21. Laurian, A., Drijfhout, S. S., Hazeleger, W. \& van den Hurk, B. Response of the Western European climate to a collapse of the thermohaline circulation. Clim. Dyn. 34, 689-697 (2010).

22. Drijfhout, S. Competition between global warming and an abrupt collapse of the AMOC in Earth's energy imbalance. Sci. Rep. 5, 14877 (2015).

23. $\mathrm{Hu}, \mathrm{A}$. et al. Role of AMOC in transient climate response to greenhouse gas forcing in two coupled models. J. Clim. 33, 5845-5859 (2020).

24. Bellomo, K., Angeloni, M., Corti, S. \& von Hardenberg, J. Future climate change shaped by inter-model differences in Atlantic meridional overturning circulation response. Nat. Commun. 12, 3659 (2021).

25. Collins, M. et al. In IPCC Special Report on the Ocean and Cryosphere in a Changing Climate (eds Pörtner, H.-O. et. al.) Ch.6 (2019).

26. Newton, R. et al. White Arctic vs. Blue Arctic: a case study of diverging stakeholder responses to environmental change. Earths Future 4, 396-405 (2016).

27. Tremblay, J.-É. et al. Current state and trends in Canadian Arctic marine ecosystems: I. Primary production. Clim. Change 115, 161-178 (2012).

28. Laidre, K. L. et al. Quantifying the sensitivity of Arctic marine mammals to climate-induced habitat change. Ecol. Appl. 18, S97-S125 (2008).

29. Huntington, H. P. et al. Evidence suggests potential transformation of the Pacific Arctic ecosystem is underway. Nat. Clim. Change 10, 342-348 (2020).

30. Rasher, D. B. et al. Keystone predators govern the pathway and pace of climate impacts in a subarctic marine ecosystem. Science 369, 1351-1354 (2020).

31. Wernberg, T. et al. Climate-driven regime shift of a temperate marine ecosystem. Science 353, 169-172 (2016).

32. Gautier, D. L. et al. Assessment of undiscovered oil and gas in the Arctic. Science 324, 1175-1179 (2009).

33. Müller, D. K. in The New Arctic (eds Evengård B., Larsen J. N. \& Paasche Ø.) Ch. 11 (Springer, 2015).

34. Fauchald, P. et al. Poleward shifts in marine fisheries under Arctic warming. Environ. Res. Lett. 16, 074057 (2021).

35. Smith, L. C. \& Stephenson, S. R. New Trans-Arctic shipping routes navigable by midcentury. Proc. Natl Acad. Sci. USA 110, E1191-E1195 (2013). 
36. Lee, $\mathrm{H}$. et al. The response of permafrost and high-latitude ecosystems under large-scale stratospheric aerosol injection and its termination. Earths Future 7, 605-614 (2019).

37. Ramage, J. et al. Population living on permafrost in the Arctic. Popul. Environ. 43, 22-38 (2021)

38. Streletskiy, D. A., Suter, L. J., Shiklomanov, N. I., Porfiriev, B. N. \& Eliseev, D. O. Assessment of climate change impacts on buildings, structures and infrastructure in the Russian regions on permafrost. Environ. Res. Lett. 14, 025003 (2019).

39. Melvin, A. M. et al. Climate change damages to Alaska public infrastructure and the economics of proactive adaptation. Proc. Natl Acad. Sci. USA 114, E122-E131 (2017).

40. Hjort, J. et al. Degrading permafrost puts Arctic infrastructure at risk by midcentury. Nat. Commun. 9, 5147 (2018)

41. Klinger, D. H., Levin, S. A. \& Watson, J. R. The growth of finfish in global open-ocean aquaculture under climate change. Proc. R. Soc. B Biol. Sci. 284, 20170834 (2017).

42. Iversen, A., Asche, F., Hermansen, Ø. \& Nystøyl, R. Production cost and competitiveness in major salmon farming countries 2003-2018. Aquaculture 522, 735089 (2020).

43. Naylor, R. L. et al. A 20-year retrospective review of global aquaculture. Nature 591, 551-563 (2021).

44. ICES. Report of the Working Group on North Atlantic Salmon (WGNAS). ICES CM 2018/ACOM:21 (2018).

45. Dunne, J. P. et al. The GFDL Earth system model version 4.1 (GFDL-ESM 4.1): overall coupled model description and simulation characteristics. J. Adv. Model. Earth Syst. 12, e2019MS002015 (2020).

46. Danabasoglu, G. et al. The community earth system model version 2 (CESM2). J. Adv. Model. Earth Syst. 12, e2019MS001916 (2020).

47. Sellar, A. A. et al. UKESM1: description and evaluation of the U.K. Earth system model. J. Adv. Model. Earth Syst. 11, 4513-4558 (2019).

48. Hajima, T. et al. Development of the MIROC-ES2L Earth system model and the evaluation of biogeochemical processes and feedbacks. Geosci. Model Dev. 13, 2197-2244 (2020).

49. Swart, N. C. et al. The Canadian Earth System Model version 5 (CanESM5.0.3). Geosci. Model Dev. 12, 4823-4873 (2019).

50. Séférian, R. et al. Evaluation of CNRM Earth System Model, CNRM-ESM2-1: role of earth system processes in present-day and future climate. J. $A d v$. Model. Earth Syst. 11, 4182-4227 (2019).

51. Ziehn, T. et al. The Australian earth system model: ACCESS-ESM1.5. J. South. Hemisph. Earth Syst. Sci. 70, 193-214 (2020).

52. O'Neill, B. C. et al. The scenario model intercomparison project (ScenarioMIP) for CMIP6. Geosci. Model Dev. 9, 3461-3482 (2016).

53. Kelley, M. et al. GISS-E2.1: configurations and climatology. J. Adv. Model. Earth Syst. 12, e2019MS002025 (2020).

54. Boucher, O. et al. Presentation and evaluation of the IPSL-CM6A-LR climate model. J. Adv. Model. Earth Syst. 12, e2019MS002010 (2020)

55. Yukimoto, S. et al. The Meteorological Research Institute earth system model version 2.0, MRI-ESM2.0: description and basic evaluation of the physical component. J. Meteorol. Soc. Jpn. Ser II 97 (2019).

56. Swingedouw, D. et al. On the risk of abrupt changes in the North Atlantic subpolar gyre in CMIP6 models. Ann. N. Y. Acad. Sci. 1504, 187-201 (2021).

57. Winton, $M$. et al. Has coarse ocean resolution biased simulations of transient climate sensitivity? Geophys. Res. Lett. 41, 8522-8529 (2014).

58. Valdes, P. Built for stability. Nat. Geosci. 4, 414-416 (2011).

59. Liu, W., Xie, S.-P., Liu, Z. \& Zhu, J. Overlooked possibility of a collapsed Atlantic Meridional overturning circulation in warming climate. Sci. Adv. 3, e1601666 (2017).

60. Bakker, P. et al. Fate of the Atlantic Meridional overturning circulation: strong decline under continued warming and Greenland melting. Geophys. Res. Lett. 43, 12,252-12,260 (2016)

61. Jones, C. D. et al. The Zero Emissions Commitment Model Intercomparison Project (ZECMIP) contribution to C4MIP: quantifying committed climate changes following zero carbon emissions. Geosci. Model Dev. 12, 4375-4385 (2019).

62. Tokarska, K. B. et al. Past warming trend constrains future warming in CMIP6 models. Sci. Adv. 6, eaaz9549 (2020).

63. Liddicoat, S. K. et al. Compatible fossil fuel CO2 emissions in the CMIP6 earth system models' historical and shared socioeconomic pathway experiments of the twenty-first century. J. Clim. 34, 2853-2875 (2021).

\section{Acknowledgements}

All authors acknowledge funding from the Research Council of Norway (project IMPOSE, grant 294930) and the Bjerknes Centre for Climate Research (project LOES). J.S. has received funding from the European Union's Horizon 2020 research and innovation program under grant agreement No 820989 (project COMFORT). The work reflects only the authors' view; the European Commission and their executive agency are not responsible for any use that may be made of the information the work contains. Supercomputing and storage resources were provided by UNINETT Sigma2 (projects nn9708k/ns9708k).

\section{Author contributions}

J.S. conceived the study, designed the model experiments, performed the model simulations, analyzed and interpreted the model data, and wrote the manuscript. A.A., N.G. and H.L. contributed to the model data analysis and interpretation and to the writing of the manuscript.

\section{Competing interests}

The authors declare no competing interests.

\section{Additional information}

Supplementary information The online version contains supplementary material available at https://doi.org/10.1038/s41467-022-28573-5.

Correspondence and requests for materials should be addressed to Jörg Schwinger.

Peer review information Nature Communications thanks Didier Swingedouw and the other, anonymous, reviewer(s) for their contribution to the peer review of this work. Peer reviewer reports are available.

Reprints and permission information is available at http://www.nature.com/reprints

Publisher's note Springer Nature remains neutral with regard to jurisdictional claims in published maps and institutional affiliations.

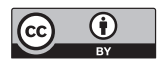

Open Access This article is licensed under a Creative Commons Attribution 4.0 International License, which permits use, sharing, adaptation, distribution and reproduction in any medium or format, as long as you give appropriate credit to the original author(s) and the source, provide a link to the Creative Commons license, and indicate if changes were made. The images or other third party material in this article are included in the article's Creative Commons license, unless indicated otherwise in a credit line to the material. If material is not included in the article's Creative Commons license and your intended use is not permitted by statutory regulation or exceeds the permitted use, you will need to obtain permission directly from the copyright holder. To view a copy of this license, visit http://creativecommons.org/ licenses/by/4.0/.

(c) The Author(s) 2022 